\title{
Comparison of Hysterosalpingography and Combined Laparohysteroscopy for the Evaluation of Primary Infertility Nigam $A,{ }^{1}$ Saxena $P,{ }^{2}$ Mishra $A^{2}$
}

${ }^{1}$ Department of Obstetrics and Gynaecology

Hamdard Institute of Medical Sciences and Research

New Delhi 110062, India.

${ }^{2}$ Lady Hardinge Medical College,

New Delhi 110001, India.

\section{Corresponding Author}

Aruna Nigam

Department of Obstetrics and Gynaecology

Hamdard Institute of Medical Sciences and Research

New Delhi 110062, India

E-mail: prakasharuna@hotmail.com

\section{Citation}

Nigam A, Saxena P, Mishra A. Comparison of Hysterosalpingography and Combined Laparohysteroscopy for the Evaluation of Primary Infertility. Kathmandu Univ Med J 2015;52(4):281-5.

\section{ABSTRACT}

\section{Background}

Hysterosalpingography (HSG) is a useful screening test for the evaluation of female infertility. Laparoscopy has proven role in routine infertility work up but role of hysteroscopy in an infertile patient with normal HSG for additional information is a subject of debate. Hysteroscopy permits direct visualization of the cervical canal and the uterine cavity and thereby helping in the evaluation of shape, and cavitary lesion.

\section{Objective}

To detect uterine abnormalities in infertile women by various approaches i.e. HSG and hysteroscopy and evaluating the role of combining hysteroscopy with laparoscopy for the evaluation of tubo-uterine factor for primary infertility.

\section{Method}

One twenty eight infertile women were evaluated and HSG was performed as a basic test for evaluation of tubes and uterine cavity. Women were subjected to combined laparoscopic and hysteroscopic examination on evidence of HSG abnormalities. In absence of any HSG abnormality, women were subjected to ovulation induction for three to six months and if they did not conceive during this period they were undertaken for combined laparo-hysteroscopic evaluation.

\section{Result}

The positive predictive value of HSG for detecting the intrauterine abnormalities was $70 \%$ among 126 patients where the hysteroscopy could be performed successfully. The diagnostic accuracy of HSG for intrauterine abnormalities revealed false negative rate of $12.96 \%$. The most frequent pathologies encountered by laparoscopy were tubal and/or peritoneal and were found in $68 \%$ (87/128) of women. Total $64.06 \%$ infertile women had some abnormality on laparoscopy. This detection rate has been increased from $64.06 \%$ to $71.86 \%$ on including the concomitant hysteroscopy.

\section{Conclusion}

HSG is a good diagnostic modality to detect uterine as well as tubal abnormalities in infertile patient. HSG and hysteroscopy are complementary to each other and whenever the patient is undertaken for diagnostic laparoscopy for the infertility, hysteroscopy should be combined to improve the detection rate of abnormalities especially in communities where there is enormous risk of pelvic infection.

\section{KEY WORDS}

Hysterosalpingography, hysteroscopy, infertility, laparoscopy 


\section{INTRODUCTION}

Infertility is one of the most prevalent health disorders in reproductive age group couples. Diagnostic assessment of infertility is indicated when pregnancy has not occurred even after one year of regular unprotected intercourse. Earlier assessment is required where the history of menstrual irregularity or male factor infertility is suspected. Major cause of female infertility is tubal pathology with a prevalence of around 30\%. ${ }^{1}$ Uterine cavity abnormalities can be the cause of infertility in $10-15 \%$ of women leading to abnormal findings in approximately $50 \%$ of infertile women. ${ }^{2}$

Imaging techniques i.e. transvaginal ultrasound and hysterosalpingography (HSG) are commonly used for the evaluation of female genital organs followed by endoscopy if required in developing countries. HSG is the most commonly used technique for the evaluation of uterine cavity i.e. anatomical abnormalities, intrauterine space occupying lesions, intrauterine adhesion and tubes i.e. tubal blockage. ${ }^{3,4}$ HSG has an advantage of being an outpatient department (OPD) procedure. It does not require any anaesthesia for the procedure but there are disadvantages like radiation exposure, exposure to iodinated contrast media and the discomfort to the patient.

During the last three decades technological advancement in the endoscopic instruments has revolutionized the management of female infertility. Laparoscopy has proven role in routine infertility work up but role of hysteroscopy in normal HSG for additional information is a subject of debate. Hysteroscopy permits direct visualization of the cervical canal and the uterine cavity and thereby helping in the evaluation of shape, cavitary lesion and vascular pattern. Hysteroscopy is traditionally being used for the evaluation of infertility where the HSG shows the abnormality. There are different thoughts regarding hysteroscopic evaluation of uterine cavity routinely. Many studies have reported inadequacy of HSG in diagnosing all intrauterine pathologies and emphasized on carrying out hysteroscopy routinely, ${ }^{5}$ whereas others have suggested selective use of hysteroscopy based on symptoms, examination and positive findings on HSG. ${ }^{6}$

Laparoscopy allows exact evaluation and treatment of tubal factor infertility and helps in deciding optimal management for an infertile patient but it requires anaesthesia and is a major operative procedure which is generally not acceptable to patients.

\section{Objective}

To detect uterine abnormalities in infertile women by various approaches i.e. HSG, hysteroscopy and laparoscopy. Evaluating the role of combining hysteroscopy with laparoscopy for the evaluation of tubo-uterine factor of primary infertility irrespective of HSG findings.

\section{METHODS}

This was a cross sectional study conducted in a tertiary care centre in North India. All the patients coming with the complaint of primary infertility from January 2011 to January 2012 in one unit were evaluated for the probable inclusion in the study. Total 128 women with primary infertility were recruited in the study after taking written informed consent. All patients were subjected to complete history regarding the duration of infertility; symptoms of genital tract infection i.e. lower abdominal pain, vaginal discharge, dyspareunia, chronic infection; coital habit; previous treatment of infertility; menstrual cycle pattern; addictions. General physical examination which included nutritional status; pallor, icterus; clubbing; thyroid gland examination; lymphadenopathy; breast examination; blood pressure and pulse rate was done in all patients. Systemic examination i.e. cardiovascular, respiratory and abdominal examination was also performed in all women. Gynaecological examination included speculum examination and bimanual vaginal examination. Cases of male factor infertility as evaluated by semen analysis and were excluded. Besides routine investigations, others such as complete blood counts, liver and kidney function tests, blood sugar, Monteux test, ultrasonography, hormonal profile was also done which included day 2 serum luteinizing and follicular stimulating hormone, thyroid stimulating hormone and serum prolactin wherever needed. Considering high prevalence of genitourinary tuberculosis in population catered, premenstrual endometrial biopsy was done for acid fast bacilli staining in every woman and polymerase chain reaction for Mycobacterium tuberculosis wherever feasible. Simultaneous histopathological examination was also done for the evidence of ovulation. Cases of active genitourinary tuberculosis were managed by anti-tubercular treatment and invasive procedures deferred for later date. Other pelvic infections were also treated before HSG and endoscopy.

HSG was performed in all women in postmenstrual phase preferably between day seven and day 10 of menstrual cycle. Approximately 5-10 ml water soluble contrast media was instilled inside the uterine cavity under fluoroscopic control. HSG abnormalities were interpreted as filling defects in the uterine cavity, uterine wall irregularities, uterine contour abnormalities and tubal contour and spillage pattern. On evidence of HSG abnormalities women were subjected to combined laparoscopy and hysteroscopy in the subsequent cycles. Where there was no evidence of abnormality, patients were subjected to ovulation induction for 3 to 6 months and if they did not conceive during that period they were undertaken for combined evaluation. Laparoscopy and hysteroscopy was performed between $7^{\text {th }}$ to $11^{\text {th }}$ days of menstrual cycle under general anaesthesia. Normal saline was used as distension media for diagnostic hysteroscopy and glycine was used only when operative procedure has to be undertaken. Hysteroscopy evaluated 
status of cervical canal, isthmus, uterine cavity and tubal ostia. Laparoscopy was performed to determine the morphology of uterus and tubes, tubo-ovarian relationship, ovarian pathology, presence of adhesion, endometriosis, fibroids or any other pathology. Chromotubation was done in all cases to assess tubal patency. Operative intervention was done wherever possible. Findings on HSG and laparohysteroscopy were compared.

Sample size estimation and statistical analysis: Sample size calculation was performed considering the success of hysterosalpingography as $70 \%$ and that of laparohysteroscopy as $90 \%$, and assuming that a $20 \%$ difference would be assumed to be significant. The alpha error was fixed at 5\% and beta error was fixed at $20 \%$. The sample size was calculated assuming these proportions and came to be 124 .

The collected data was tabulated on a microsoft excel worksheet on window based operating system. The hysteroscopic and laparoscopic findings were used as a reference standard to calculate sensitivity, specificity, positive predictive values (PPV) and false-negative rates of HSG.

\section{RESULTS}

Total 128 infertile women were recruited in the study, with age group ranging from 20 years to 33 years. The duration of infertility varied from 3 years to 16 years. Significant past history of genitourinary tuberculosis was given by $9.38 \%$ $(n=12)$ women and $39.06 \% \quad(n=50)$ women had either history or treatment suggestive of past pelvic infection. Thus $50 \%$ of women had some factor suggestive of pelvic infection before performing HSG and endoscopy for which they have been given antibiotic treatment before the procedure.

Laparoscopy was successfully done in all 128 women. The hysteroscopy could be performed in 126 (98.44\%) cases and had to be abandoned in two cases due to perforation giving failure rate of $1.56 \%$. As multiple lesions were detected in some patients during HSG/laparoscopy or hysteroscopy which are expressed individually, the total number of detected abnormalities exceeds the actual number of the patients.

HSG abnormalities were present in 78.1\% (100/128) women. Most of the abnormalities were present in the form of blocked tubes in 96 cases (76\%) either unilateral (37.50\%) or bilateral $(62.50 \%)$ or cornual $(41.66 \%)$ or fimbrial $(27.08 \%)$ or combination $(31.25 \%)$ of it. This is followed by hydrosalpinx in $15.60 \%$ women, endometritis in $7.80 \%$ women, beaded tubes in $3.12 \%$ women and intrauterine adhesions in $1.56 \%$ women. There was evidence of uterine anomaly in $4.68 \%$ cases $(n=6)$ in the form of unicornuate uterus, bicornuate uterus and arcuate uterus. 64.5\% cases showed the tubal blockade on laparoscopy similar to HSG. Twenty eight patients who had no abnormality on HSG and when they did not conceive after ovulation induction were taken for laparohysteroscopy. In these patients 18 have normal laparohysteroscopy, six patients had fimbrial adhesions and loculated spill from fallopian tubes, three have flimsy peritoneal adhesions and one patient has endometrial polyp on hysteroscopy. Refer Table 1 for the hysteroscopic abnormalities. As mentioned in methodology the endoscopic findings were taken as reference standards, the diagnostic accuracy of HSG for intrauterine abnormalities revealed false negative rate of $12.96 \%$. The positive predictive value of HSG for detecting the intrauterine abnormalities was $70 \%$.

\section{Table 1. Hysteroscopic abnormalities}

\begin{tabular}{|lll|}
\hline Abnormality & Number of women & $\%$ \\
\hline Adhesive pathology [Total] & 18 & 14.28 \\
\hline $\begin{array}{l}\text { 1. Adhesions in Uterine Cavity } \\
\begin{array}{l}\text { 2. Adhesions at Utero Tubal } \\
\text { Junction }\end{array}\end{array}$ & 6 & 4.76 \\
\hline $\begin{array}{l}\text { 3 Adhesions at cervico-isthemic } \\
\text { junction }\end{array}$ & 2 & 7.93 \\
\hline $\begin{array}{l}\text { Uterine polyp } \\
\text { Endometritis }\end{array}$ & $\mathbf{2}$ & 1.58 \\
\hline Uterine malformation [Total] & 4 & 1.58 \\
\hline
\end{tabular}

Table 2 delineates the laparoscopic findings of 128 infertile women. The most frequent pathologies encountered by laparoscopy were tubal and/or peritoneal, believed to be post-inflammatory and were found in up to $68 \%$ of infertile women. HSG findings regarding normal tubes were in agreement in 18 women on laparoscopy and 60 tubal occlusion cases. Thus sensitivity of HSG in diagnosing the tubal occlusion correctly was $62.6 \%$ and specificity was $50 \%$. Laparoscopy revealed some abnormal finding in $50 \%$ of infertile women. $14.06 \%$ infertile women showed abnormalities both on laparoscopy and hysteroscopy. Hysteroscopy detected uterine cavity abnormalities in $7.8 \%$ women who had normal laparoscopy. Thus, the detection rate of abnormalities has been increased from $64.06 \%$ to $71.86 \%$ on including the simultaneous hysteroscopy.

Table 2. Laparoscopic abnormalities

\begin{tabular}{|lll|}
\hline Laparoscopic abnormality & $\begin{array}{l}\text { Number of women } \\
(\mathbf{N}=\mathbf{1 2 8})\end{array}$ & $\%$ \\
\hline Dense adhesions & 52 & 40.60 \\
\hline Flimsy adhesion & 12 & 9.30 \\
\hline Endometriosis & 16 & 12.50 \\
\hline Hydrosalpinx & 24 & 18.75 \\
\hline Unilateral tubal block & 27 & 21.09 \\
\hline Bilateral tubal block & 33 & 25.70 \\
\hline Convoluted tubes & 6 & 4.68 \\
\hline Fibroid uterus & 4 & 3.12 \\
\hline Tubo-ovarian mass & 4 & 3.12 \\
\hline Uterine malformation & 4 & 3.12 \\
\hline
\end{tabular}




\section{DISCUSSION}

Evolution in the availability of diagnostic techniques has changed the infertility management strategies in the current era. But still the cost and the availability in the developing countries restricts their free use in general population. Importance of diagnosis of the cause of infertility cannot be denied for successful management of infertility. The careful selection of patients based on clinical history, physical examination and appropriate investigative modality will identify those patients who will benefit from endoscopic examination for their infertility evaluation.

In literature review, there is varied detection rate regarding laparoscopic and hysteroscopic evidences of abnormalities in women with primary infertility. In present study laparoscopy has detected significant abnormality accounting for infertility in $64.06 \%$ infertile women which is similar to other studies. ${ }^{7}$ Studies from different part of world had reported hysteroscopic abnormalities ranging from $8 \%$ to $40 \%$ in infertile women. ${ }^{8}$ According to literature approximately one fourth of women attending infertility clinics have some intrauterine abnormality which is responsible for failure of implantation. ${ }^{9}$ In our study hysteroscopy was able to detect significant abnormality in $22.22 \%$ women of primary infertility which is comparable to few other studies where they have reported detection rate of $32 \%-34 \% .{ }^{8,10}$ Variation could be due to difference in population studied and subjective variation in interpretation of hysteroscopic findings. Women with hysteroscopic abnormality are lesser in our study as we have included only those lesions which are definitely related to infertility and excluded lesions such as endometrial hyperplasia, petechiae etc as some researchers raised doubts on significance of all hysteroscopic abnormalities as a etiology of infertility. ${ }^{9}$ The study by Preutthipan et al. had reported hysteroscopic abnormalities in $46 \%$ cases with most common abnormality to be intrauterine adhesion in $22 \%$ cases and endometrial polyp in $16 \%$ cases. ${ }^{11}$ They have also revealed $98.0 \%$ sensitivity, $34.9 \%$ specificity, PPV $69.9 \%$ and NPV $92.0 \%$ with HSG in the detection of intrauterine pathology. ${ }^{11}$ In our study also the most common intrauterine pathology was adhesion in $14 \%$ cases which is comparable but endometrial polyp was present in only $2 \%$ cases in our study. In our study the PPV of HSG for detecting the intrauterine abnormalities was $70 \%$ and NPV of $87 \%$ which was comparable.

In present study $7.8 \%$ women had abnormalities only on hysteroscopy while in study conducted by Shokeir et al. $12.3 \%$ women had significant lesion on hysteroscopy with normal laparoscopic findings. ${ }^{7}$ In present study omission of hysteroscopy would have missed etiology in $7 \%$ cases of infertility same as reported by Saridogan et al. ${ }^{8}$
The sensitivity of HSG in diagnosing the tubal occlusion correctly was $62.6 \%$ in our study. The sensitivity of HSG in diagnosing tubal occlusion is varies from $65 \%$ to $96 \%$ in different studies. ${ }^{12}$ The possible cause of difference in the diagnosis of tubal occlusion between HSG and laparoscopy might be the use of anaesthesia laparoscopic examination which relieves the tubal spasm. The other important factor is the time interval between the two procedures as with the continuation of the time interval the conditions are made for the aggravation of old and occurrence of new pathological processes in genital tract.

In present study peritoneal and tubal pathology is the most commonly detected abnormality during laparoscopy. In this study $49.9 \%$ patients had adhesions (thick + filmsy) on laparoscopy, 32.8\% patients had tubal pathology and 3.12\% had tubo-ovarian masses. In a study from Trivendrum, India, laparoscopy in infertility revealed significant pelvic pathology in $26.8 \%$ cases. $^{13}$ An another study from developing countries showed equal prevalence of pelvic adhesion and tubal pathology that was $40 \%$ in primary infertility. ${ }^{9}$ This could be due to of higher prevalence of pelvic infection in the developing countries.

\section{Limitations}

As both the test HSG and the laparo-hysteroscopy have not been done simultaneously it might be possibility that the disease may have progressed, which can give positive result. The HSG and the laparo-hysteroscopy have not been done by the same operator which may give a bias although the HSG films have been read by the same operator who had done the laparo-hysteroscopy.

\section{CONCLUSION}

HSG is a useful test for initial evaluation of infertile female. The HSG actually suspects the lesion and hysteroscopy has an advantage of directly visualizing it. As the HSG provides valuable information regarding the uterus and the tubes, it is recommended to be primary screening test for the evaluation of infertility. Whenever intrauterine abnormality is detected, patient must undergo hysteroscopic evaluation, moreover if the patient is undertaken for diagnostic laparoscopy for the infertility, hysteroscopy should be combined to improve the detection rate of abnormalities irrespective of normal HSG findings. Combined approach allows prompt and complete identification of all contributory factors, helping the gynaecologist to institute appropriate therapy and will help in higher conception rates. As there are high rates of tubal abnormalities in the infertile patients and the patients do not conceive even after normal evaluation of the tubes, the further research can be done on the hysteroscopic evaluation of the tubal mucosa. 


\section{REFERENCES}

1. Evers JL. Female subfertility. Lancet 2002:360;151-9.

2. Brown SE, Coddington CC, Schnorr J, Toner JP, Gibbons W, Oehninger S. Evaluation of outpatient hysteroscopy, saline infusion hysterosonography, and hysterosalpingography in infertile women: a prospective, randomized study. Fertil Steril 2000;74:1029-34.

3. Tsuji I, Ami K, Fujinami $\mathrm{N}$, Hoshiai $\mathrm{H}$. The significance of laparoscopy in determining the optimal management plan for infertile patients with suspected tubal pathology revealed by hysterosalpingography. Tohoku J Exp Med 2012;227:105-8.

4. Roma Dalfo, Ubeda B, Ubeda A, Monzon M, Rotger R, Ramos R,et al. Diagnostic value of hysterosalpingography in the detection of intrauterine abnormalities: a comparison with hysteroscopy. AJR Am J Roentgenol 2004;183:1405-9.

5. Cicinelli E, Matteo M, Causio F, Schonauer LM, Pinto V, Galantino P. Tolerability of the mini-pan-endoscopic approach (transvaginal hydrolaparoscopy and minihysteroscopy) versus hydrosalpingography in an outpatient infertility investigation. Fertil Steril 2001;76:1048-51.

6. Crosignani PG, Rubin BL. Optimal use of infertility diagnostic tests and treatments. The ESHRE Capri Workshop Group. Hum Reprod 2000;15:723-32.
7. Shokeir TA, Shalan HM, El-Shafei MM. Combined diagnostic approach of laparoscopy and hysteroscopy in the evaluation of female infertility: Results of 612 patients. J Obstet Gynaecol Res. 2004;30:9-14.

8. Levi Setti PE, Colombo GV, Savasi V, Bulletti C, Albani E, Ferrazzi E. Implantation failure in assisted reproduction technology and a critical approach to treatment. Ann N Y Acad Sci 2004;1034:184-99.

9. Hucke J, De Bruyne F, Balan P. Hysteroscopy in infertility-diagnosis and treatment including falloposcopy. Contrib Gynecol Obstet 2000;20:13-20.

10. Saridogan E, Bolaji I I, Boto T, Djahanbakhch O. Experience with combined use of laparoscopy and hysteroscopy for the investigation of infertility. Gynaecological Endoscopy 1997;6:119-23.

11. Preutthipan S, Linasmita V. A prospective comparative study between hysterosalpingography and hysteroscopy in the detection of intrauterine pathology in patients with infertility. J Obstet Gynaecol Res 2003;29:33-7.

12. Bacevac J, Ganovic R. Diagnostic value of hysterosalpingography in examination of Fallopian tubes in infertile women. Srp Arh Celok Lek 2001;129:18-21.

13. Jayakrishnan K, Koshy AK, Raju R. Role of laparohysteroscopy in women with normal pelvic imaging and failed ovulation stimulation with intrauterine insemination. J Hum Reprod Sci. 2010;3:20-24. 